\title{
Active magneto-plasmonics in hybrid metal-ferromagnet structures
}

\author{
Vasily V. Temnov^, Gaspar Armelles², Ulrike Woggon³, Dmitry Guzatov, Alfonso Cebollada², \\ Antonio Garcia-Martin2 ${ }^{2}$ Jose-Miguel Garcia-Martin², Tim Thomay5 ${ }^{5}$, Alfred Leitenstorfer ${ }^{5}$ \\ and Rudolf Bratschitsch ${ }^{5}$
}

Surface-plasmon-mediated confinement of optical fields holds great promise for on-chip miniaturization of all-optical circuits ${ }^{1-4}$. Following successful demonstrations of passive nanoplasmonic devices ${ }^{5-7}$, active plasmonic systems have been designed to control plasmon propagation. This goal has been achieved either by coupling plasmons to optically active materials ${ }^{8-13}$ or by making use of transient optical nonlinearities in metals via strong excitation with ultrashort laser pulses ${ }^{14-17}$. Here, we present a new concept in which the active optical component is a metal-ferromagnet-metal structure. It is based on active magneto-plasmonic microinterferometry, where the surface plasmon wave vector in a gold-ferromagnet-gold trilayer system is controlled using a weak external magnetic field. Application of this new technique allows measurement of the electromagnetic field distribution inside a metal at optical frequencies and with nanometre depth resolution. Significant modulation depth combined with possible all-optical magnetization reversal induced by femtosecond light pulses ${ }^{18}$ opens a route to ultrafast magneto-plasmonic switching.

Continuous improvements in nanofabrication and nanocharacterization capabilities have changed projections about the role that metals could play in the development of new optical devices. Our present ability to manipulate both the electromagnetic field localization and the nanoscale coupling between light and surface plasmons leads to the observation of new optical phenomena such as enhanced optical transmission, sub-wavelength confinement of optical fields and sub-wavelength optical resolution.

A magnetic field can modify the properties of an electron plasma. This effect has been analysed for surface plasmons at terahertz frequencies propagating at an interface between a semiconductor and a dielectric $^{19}$ (also known as surface plasmon polariton, SPP). In particular, if the magnetic field is applied parallel to the interface and perpendicular to the SPP wave vector, it induces a modification of the SPP wave vector while keeping its transverse magnetic (TM) character. Therefore, the magnetic field could be used to control SPP propagation, opening the door for novel active plasmonic devices. The technological drawback is that the magnetic fields that enable noticeable changes in noble-metal-based plasmonic structures in the visible spectral range are of the order of several tesla and are therefore too large for realistic applications.

Ferromagnetic layers, however, exhibit a large magneto-optical (MO) activity, but do not support propagating long-range surface plasmons due to high ohmic losses. Combining magnetic and plasmonic counterparts in a magneto-plasmonic system would both enable high MO activity and support propagating surface plasmon modes. Orders of magnitude smaller external magnetic fields might be sufficient to manipulate the optical properties of their SPPs ${ }^{20}$. This concept allows the design and tailoring of the optical properties of complex composite magnetic materials ${ }^{21-24}$ towards particular potential applications, such as high-sensitivity biosensing devices ${ }^{25}$.

Here, we use plasmonic microinterferometry, $92,17,26,27$ to demonstrate active control of surface plasmons in a magneto-plasmonic gold-cobalt-gold multilayer film by a weak external magnetic field of a few millitesla. For a gold-cobalt-gold multilayer film with a cobalt thickness of a few nanometres, the magnetization along the easy axes (in the plane of the film) may be saturated by applying a very low magnetic field of the order of $10 \mathrm{mT}$. If the magnetic field is applied along the $x$-axis (see geometry in Fig. 1), the bulk dielectric tensor of cobalt,

$$
\epsilon_{\mathrm{Co}}=\left(\begin{array}{ccc}
\epsilon_{x x} & 0 & 0 \\
0 & \epsilon_{x x} & \epsilon_{y z} M_{x} \\
0 & -\epsilon_{y z} M_{x} & \epsilon_{x x}
\end{array}\right)
$$

is governed by the magnetization $M_{x}$ in the same direction. Thus, in the composite gold-cobalt-gold films the properties of surface plasmons should not depend directly on the external magnetic field $B$, but on the magnetization $M_{x}$ in a cobalt layer. The wave vector of the gold-cobalt-gold film surface plasmon $k_{s p}\left(M_{x}, h\right)=k_{\mathrm{sp}}^{0}+$ $\Delta k_{\mathrm{mp}}\left(M_{x}, h\right)$ propagating in the $y$-direction depends on the component of the magnetization $M_{x}$ of the cobalt layer and the depth $h$ of the cobalt layer below the gold/air interface. Analytical thin-film calculations in gold-cobalt-gold multilayer films (see Supplementary Information for a detailed discussion) in the approximation of an infinitely thin cobalt layer demonstrate the possibility of magnetically modulating the surface plasmon wave vector,

$$
\Delta k_{\mathrm{mp}}\left(M_{x}, h\right) \simeq \frac{2 h_{1}\left(k_{0} \epsilon_{\mathrm{Au}} \epsilon_{\mathrm{Air}}\right)^{2}}{\left(\epsilon_{\mathrm{Air}}+\epsilon_{\mathrm{Au}}\right)\left(\epsilon_{\mathrm{Air}}^{2}-\epsilon_{\mathrm{Au}}^{2}\right.} \frac{i \epsilon_{y z}}{\epsilon_{x x}} M_{x} \exp \left(-h / \delta_{\text {skin }}\right)
$$

which scales linearly with $M_{x}$ and exponentially decays as a function of $h$ within a skin depth of light $\delta_{\text {skin }}\left(h_{1}\right.$ is the cobalt layer thickness). The maximum possible modulation of the surface plasmon wave vector $2 \Delta k_{\text {sap }}(h)=k_{\mathrm{sp}}\left(M_{x}=+1, h\right)-k_{\mathrm{sp}}\left(M_{x}=-1, h\right)$ can be achieved upon magnetization switching $\left(M_{x}= \pm 1\right.$ for a saturated sample).

Numerical calculations not only justify the validity of equation (2) for $h_{1} \ll \delta_{\text {skin }}$, but also prove that the presence of a thin cobalt-layer introduces only a minor perturbation in the electric

'Department of Chemistry, Massachusetts Institute of Technology, Cambridge, Massachusetts 02139, USA, ${ }^{2}$ Instituto de Microelectrónica de Madrid-IMM (CNM-CSIC), 28760 Tres Cantos, Madrid, Spain, ${ }^{3}$ Institut für Optik und Atomare Physik, TU Berlin, Strasse des 17. Juni 135, 10632 Berlin, Germany, ${ }^{4}$ Yanka Kupala Grodno State University, 230023 Grodno, Belarus, ${ }^{5}$ Department of Physics and Center for Applied Photonics, University of Konstanz, D-78457 Konstanz, Germany. *e-mail: temnov@mit.edu 


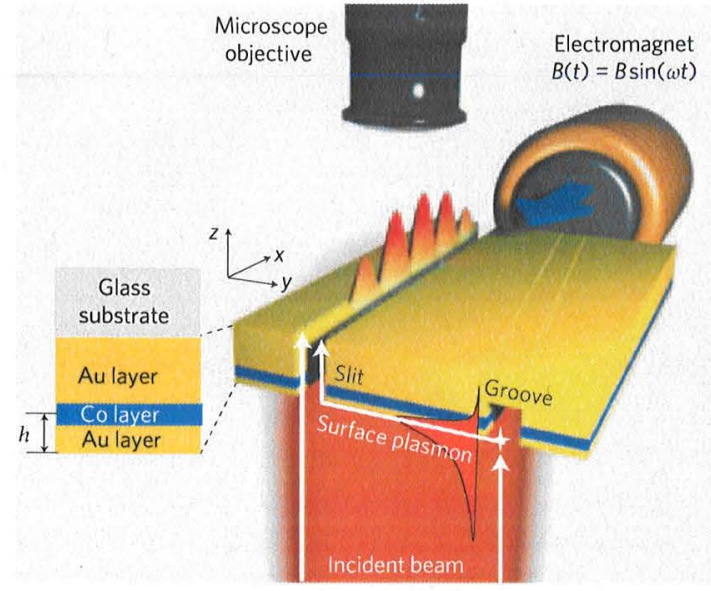

Figure 1 | Active magneto-plasmonic interferometry. A plasmonic microinterferometer consisting of a tilted slit-groove pair is milled in a goldcobalt-gold multilayer film using a focused ion beam. Surface plasmons are launched by the groove, propagate towards the slit, and interfere with the directly transmitted light to produce a periodic interference pattern along the slit axes. The oscillating magnetic field of an electromagnet is used to periodically switch the magnetization in the thin cobalt layer and thus modify the wave vector of surface plasmons. An imaging nano-optical set-up is used to record the magneto-plasmonic modulation signal.

field distribution inside the metal as compared to bulk gold (see Supplementary Fig. 1S). These calculations suggest that the dependence of the magnetic modulation of the surface plasmon wave vector as a function of cobalt layer position $h$ may be used to directly measure the skin depth of light in gold at optical frequencies with a spatial resolution determined by the thickness $h_{1}$ of the cobalt layer.

To explore the modulation of the surface plasmon wave vector, the gold-cobalt-gold multilayer films were patterned with plasmonic microinterferometers (Fig. 1). They consisted of a tilted slitgroove pair milled by a focused ion beam in a gold-cobalt-gold film grown on a glass substrate. The length of both the slit and the groove was $50 \mu \mathrm{m}$. The slit had a width of $100 \mathrm{~nm}$. Grooves were $100 \mathrm{~nm}$ deep and $200 \mathrm{~nm}$ wide. The slit-groove tilt angle $\Theta$ was varied between $5^{\circ}$ and $10^{\circ}$ and the minimum slit-groove distance $d_{0}$ varied from 0 to $20 \mu \mathrm{m}$. Illumination of a microinterferometer with a collimated $p$-polarized continuous-wave laser diode at normal incidence $(100 \mathrm{~mW}, 808 \mathrm{~nm}$, spot diameter $30 \mu \mathrm{m}$ FWHM) excited surface plasmons, which were launched by the groove. They propagated towards the slit, where they were reconverted into free-space radiation, interfering with light directly transmitted through the slit (Fig. 1). Owing to the slit-groove tilt angle, the geometrical path difference $d(x)=d_{0}+x \sin \Theta$ acquired by surface plasmons on the way to the slit changes linearly along the slit axis $x$, resulting in a sinusoidal interference pattern $I(x, B=0)=E_{1}(x)^{2}+E_{2}(x)^{2}+2 E_{1}(x) E_{2}(x) \cos (\Phi(x))$ with the two electric fields $E_{1}(x)$ and $E_{2}(x)$ and the phase $\Phi(x)=k_{\mathrm{sp}} d(x)+\phi_{0}$. An oscillating external magnetic field with amplitude $B$ was applied in the $x$-direction to periodically switch the magnetization of the cobalt layer and modulate the surface plasmon wave vector. In that way, the phase and the contrast of the plasmonic interferogram changed. A lock-in based scanning imaging optical set-up ${ }^{17}$ allowed the plasmonic interferogram to be recorded at zero magnetic field $I(x, B=0)$ together with the magneto-plasmonic interferogram $I_{\mathrm{mp}}(x, B)=I(x,+B)-I(x,-B)$ for a weak external magnetic field of a few tens of millitesla. A normalization procedure was applied to compensate for the spatially inhomogeneous modulation depth of $I(x)$ (which is equal to $4 E_{l}(x) E_{2}(x)$, marked as the grey area in Fig. 2) to obtain the normalized magneto-plasmonic interference pattern

$$
I_{\mathrm{mp}}^{(\mathrm{norm})}(x, B)=-d(x)\left|\Delta k_{\mathrm{mp}}\right| \cos (\Phi(x)+\phi)
$$

Interferograms for two different plasmonic microinterferometers defined in a gold-cobalt-gold $(5 \mathrm{~nm} / 6 \mathrm{~nm} / 189 \mathrm{~nm})$ multilayer film on glass are shown in Fig. 2. The microinterferometers have the same slit-groove tilt angle $\Theta=5^{\circ}$, but differ in the initial distance $d_{0}\left(d_{0}=0\right.$ and $\left.d_{0}=10 \mu \mathrm{m}\right)$. Both interference patterns $I(x)$ and $I_{\mathrm{mp}}(x)$ have the same period $\lambda_{\mathrm{sp}} / \sin \Theta$, but are shifted with respect to each other by a constant phase $\phi \simeq 70^{\circ}$. A change in absorption of the surface plasmons only affects the contrast of the plasmonic interference $I(x)$; that is, $I_{\mathrm{mp}}(x)$ is in phase $(\phi=0)$. A change in the surface plasmon wave vector, however, results in a fringe shift of $I(x)$; that is, $I_{\mathrm{mp}}(x)$ is shifted by $\phi=\pi / 2$. The observed phase shift $\phi \simeq 70^{\circ}$ is close to $\pi / 2$, which clearly demonstrates that the magneto-plasmonic signal is dominated by the change of the real part of the surface plasmon wave vector.

Figure $2 \mathrm{~g}$, h shows the normalized magneto-plasmonic signals $I_{\mathrm{mp}}^{(\mathrm{norm})}(x)$ described by equation(3) for an oscillating magnetic field with an amplitude of $20 \mathrm{mT}$. As expected, the amplitude $\left|\Delta k_{\text {mp }}\right| d(x)$ of the normalized magneto-plasmonic interferogram $I_{\mathrm{mp}}^{(\text {norm })}(x)$ scales linearly with surface plasmon propagation distance $d(x)=d_{0}+x \sin \Theta$ between the slit and the groove, visualized by straight dashed lines. The intrinsically small magneto-plasmonic modulation of the surface plasmon wave vector $\left|\Delta k_{\mathrm{mp}}\right| / k_{0} \approx 10^{-4}$ is accumulated over the long propagation distance, resulting in noticeable values of the magneto-plasmonic signal. In that way, a substantial enhancement factor of $k_{0} d \approx 100$ is achieved. For the central part of the microinterferometer in Fig. $2 b$ with $d=12 \mu \mathrm{m}$, we obtain a modulation depth of $2\left|\Delta k_{\mathrm{mp}}\right| d \simeq 0.01$ (the factor 2 accounts for magnetization switching between $M_{x}=-1$ and $\left.M_{x}=+1\right)$. The experimental data for a microinterferometer with a larger distance $d=22 \mu \mathrm{m}$ (interferograms not shown) provide a value $2\left|\Delta k_{\mathrm{mp}}\right| d=0.02$, which is promising for potential applications such as an optical switch. The slit-groove spacing of a few tens of micrometres needed to achieve a significant modulation depth of the magneto-plasmonic signal limits the minimum device size. At the same time, a long propagation distance is favourable for sensing applications. A further increase of the slit-groove spacing results in a reduced modulation depth of $I(x)$ due to larger surface plasmon propagation losses.

To explore the physical mechanism that links the external magnetic field with the interference pattern, the magneto-plasmonic signal $\left|\Delta k_{\mathrm{mp}}(B)\right| d$ is plotted against magnetic field amplitude $B$ in Fig. 3. A gradual increase of the signal at small fields is observed, followed by a sudden jump at $12 \mathrm{mT}$ and saturation behaviour above $15 \mathrm{mT}$. This result indicates that the magneto-plasmonic signal does not directly depend on the external magnetic field in a linear way, but is governed by the cobalt layer magnetization. This effect is demonstrated in the inset, where the magneto-plasmonic hysteresis loop (reconstructed from the temporal dependence of $\Delta k_{\mathrm{mp}}(t)$ and $\left.B(t)\right)$ is depicted together with the transverse Kerr effect hysteresis loop measured in an unpatterned sample. The cycles have identical shapes, confirming the direct dependence of the magneto-plasmonic signal on the cobalt magnetization.

Up to this point, we have demonstrated control of the surface plasmon wave vector by a small external magnetic field. We now show that this effect allows for local probing of the electromagnetic field distribution inside the gold layer. For this purpose we have performed magneto-plasmonic interferometry in multilayer structures where the cobalt layer is located at different depth $h$ below the goldair interface. In Fig. 4 we compare the results of magneto-plasmonic measurements obtained for $d=22 \mu \mathrm{m}$ with the theoretical formula 

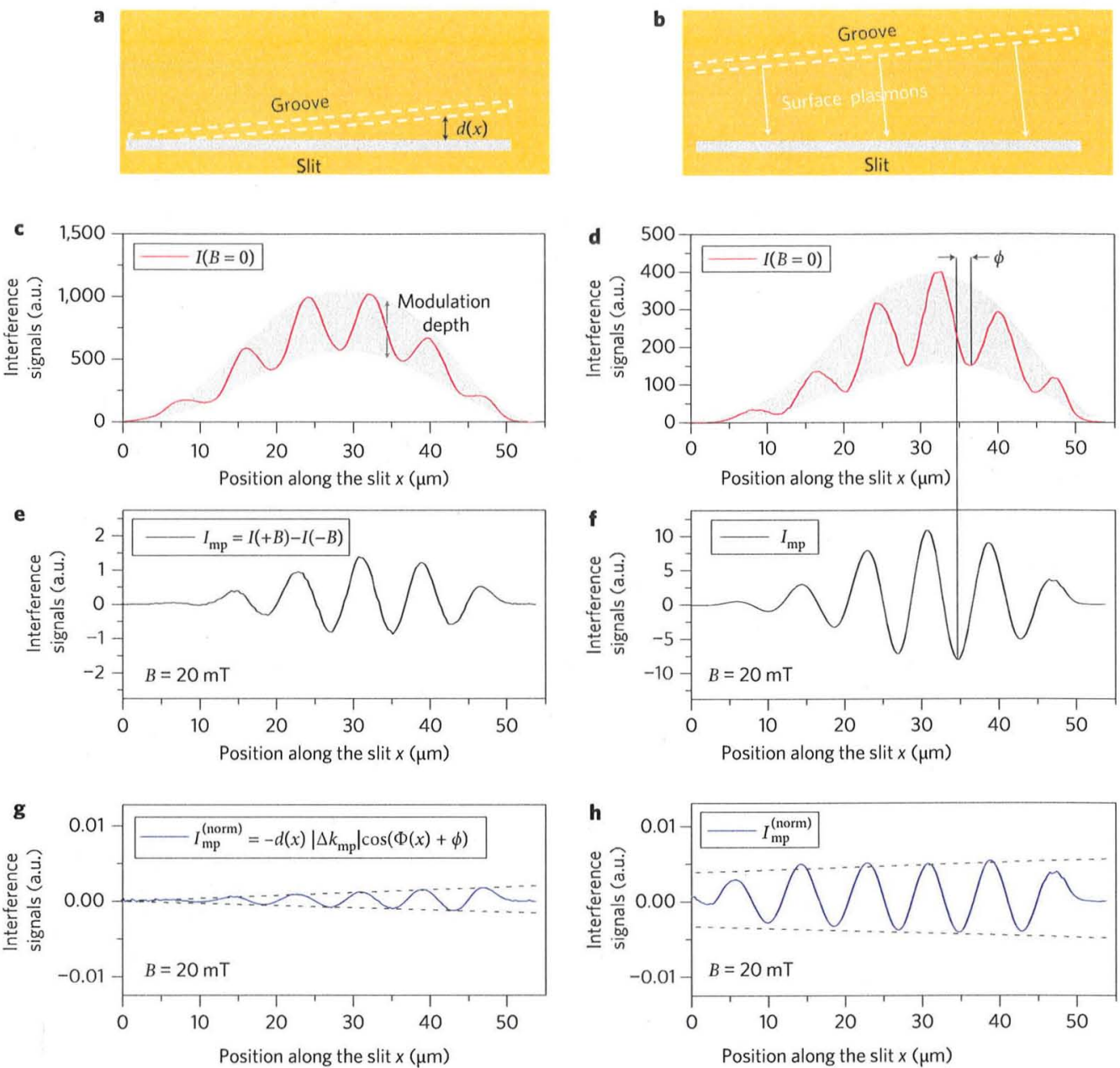

Figure 2 | Magneto-plasmonic interferograms in tilted slit-groove microinterferometers. a,b, Two plasmonic microinterferometers with the same tilt-angle of $\Theta=5^{\circ}$, but different minimum slit-groove spacings: $d_{0}=0$ (a) and $d_{0}=10 \mu \mathrm{m}(\mathbf{b})$. c, d, The plasmonic interference pattern $I(x, B=0)$ at zero magnetic field is due to surface plasmons propagating from the groove towards the slit. e,f, The periodic cycling of the magnetic field between $+20 \mathrm{mT}$ and $-20 \mathrm{mT}$ changes the surface plasmon wave vector, shifts the fringes of $I(x, B)$ and gives rise to the magneto-plasmonic signal $I_{\mathrm{mp}}(x)=I(+B)-I(-B)$. $\mathrm{g}, \mathrm{h}, \mathrm{After}$ normalization to the spatially inhomogeneous modulation depth of the plasmonic interferogram (grey shaded area in $\mathbf{c}, \mathrm{d}$ ) the normalized pattern $I_{\mathrm{mp}}^{(\text {norm })}(x)$ shows the magneto-plasmonic modulation $\left|\Delta k_{\text {mp }}\right|$ of the surface plasmon wave vector. The magnitude of the magneto-plasmonic signal $\left|\Delta k_{\mathrm{mp}}\right| d(x)$ scales linearly with slit-groove distance $d(x)$.

given by equation (2). Excellent agrement between theory and experiment is obtained without using any fit parameters. The magneto-plasmonic signal decays exponentially within the skin depth of $13 \mathrm{~nm}$. This quantitative agreement demonstrates the ability of the new experimental method to directly measure the skin depth of light inside a metal at optical frequencies with nanometre resolution. It also reveals the potential of equation (2) for the engineering of magneto-plasmonic devices. For example, a strong enhancement of magneto-plasmonic modulation could be achieved by increasing the dielectric constant of the upper dielectric material.

To explore the potential switching speed of our magnetoplasmonic devices, local generation of magnetic fields by surface currents with integrated on-chip electric circuits may be applied. Using magnetic pulses with appropriate characteristics, a precession-driven magnetic switching in the gigahertz range can be achieved ${ }^{28}$. The ultimate speed limits of this technique could be explored by applying currents driven with intense picosecond terahertz pulses and using ultrafast coherent control of magnetization by femtosecond lasers $^{29}$. A possible route towards switching at terahertz rates has been demonstrated recently. Stanciu et al. have shown that the magnetization in GdFeCo can be reversed in a reproducible manner with a single circularly polarized 40 fs laser pulse focused to a $20 \mu \mathrm{m} \mathrm{spot}{ }^{18}$. The origin of this effect is still subject to debate and may be governed by the interplay of different non-equilibrium processes. Nevertheless, these results are promising for femtosecond all-optical magnetization switching.

In summary, we have demonstrated magnetic field control of surface plasmons in a composite gold-cobalt-gold multilayer film by magneto-plasmonic interferometry. Significant phase shifts are achieved with external magnetic fields as small as a few millitesla. Accurate measurements of the skin depth of visible light in gold in the optical frequency range open the way for nanometre-resolved measurements of optical fields and magneto-plasmonic properties of matter within the skin depth of nanostructured metal/ferromagnet composites. The modulation of plasmonic optical properties using magnetization control in ferromagnetic materials by relatively weak magnetic fields suggests a straightforward application of this system as optical switches. The magnitude of magneto-plasmonic effects may be further enhanced through nanostructuring of metal composites, for example, with sub-wavelength hole arrays ${ }^{30}$. Motivated by 


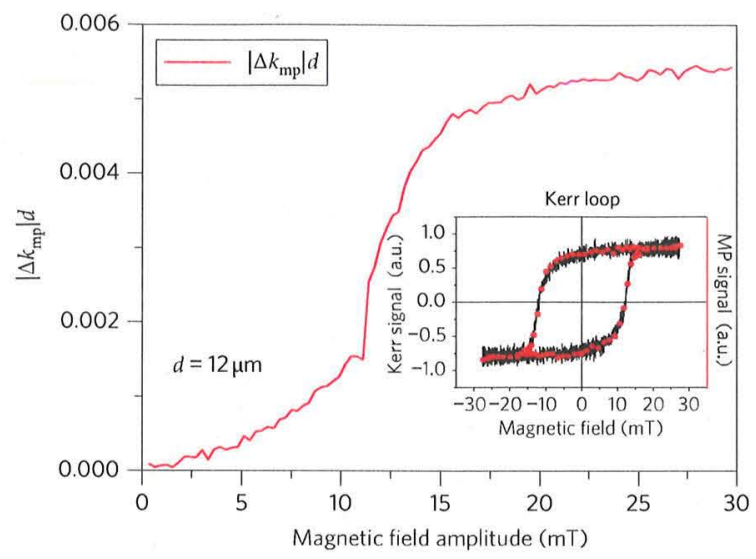

Figure 3 | Magnetization switching of the magneto-plasmonic signal. The magnitude of the magneto-plasmonic signal $\left|\Delta k_{\text {mp }}\right| d$ shows a pronounced threshold behaviour around $B=12 \mathrm{mT}$. The inset shows how the magnetoplasmonic hysteresis loop (dots) coincides with the Kerr loop measured in the transversal configuration (line).

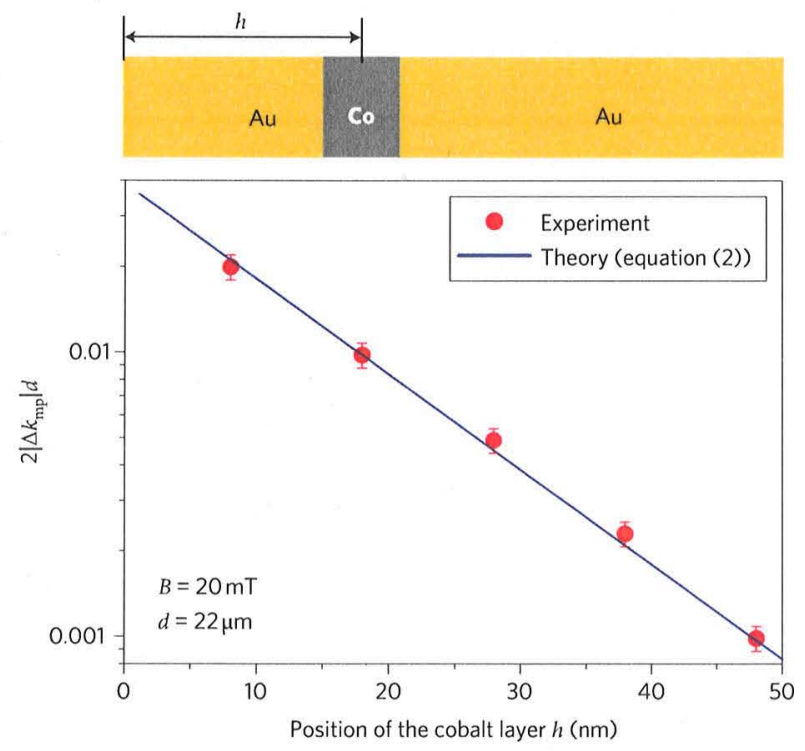

Figure 4 | Probing the electromagnetic field inside the gold layer. The measured magnitude of the normalized magneto-plasmonic signal as a function of the cobalt position $h$ shows a mono-exponential decay within the skin depth of $13 \mathrm{~nm}$, in agreement with the fit-free theoretical expression given by equation (2). The $10 \%$ error bars shown here are related to uncertainties in the groove-to-slit distance, $d$, and in the numerical calculation of modulation depth.

recent experiments with femtosecond light pulses ${ }^{18}$, we believe that the switching speed of our device could eventually reach the terahertz regime.

\section{Methods}

Fabrication of magneto-plasmonic microinterferometers. Metal deposition on glass substrates was carried out by d.c. magnetron sputtering at $20 \mathrm{~W}$ power in an ultrahigh-vacuum chamber with a base pressure in the low $10^{-9} \mathrm{mbar}$ range. The substrates were previously ultrasound-cleaned in successive baths of acetone, methanol and deionized water. Once loaded into the deposition chamber, the substrates were outgassed for $30 \mathrm{~min}$ at $150^{\circ} \mathrm{C}$. After cooling to room temperature, all layers were deposited. First, a 2-nm-thick chromium buffer layer was grown to improve adhesion of the successive layers. Then, gold-cobalt-gold trilayers with a total thickness of $200 \mathrm{~nm}$ were deposited. The position $h$ of the 6 -nm thin cobalt layer varied in five samples between $8 \mathrm{~nm}$ and $48 \mathrm{~nm}$ below the gold-air interface.
Argon deposition pressure and deposition rates were $1 \times 10^{-3} \mathrm{mbar}$ and $12 \mathrm{~nm} \mathrm{~min}^{-1}$ for gold, $3 \times 10^{-3}$ mbar and $2 \mathrm{~nm} \mathrm{~min}^{-1}$ for chromium, and $6.6 \times$ $10^{-3}$ mbar and $2.8 \mathrm{~nm} \mathrm{~min}^{-1}$ for cobalt. Plasmonic microinterferometers were milled into the multilayers with a $30-\mathrm{kV} \mathrm{Ga}^{+}$focused ion beam.

Measurements. The magnetization in the cobalt layer was switched at a frequency of $690 \mathrm{~Hz}$ by applying a periodic magnetic field with an amplitude of tens of millitesta in a resonantly driven electromagnet coil of a transformer, which was embedded in a series LC-circuit (quality factor $Q \simeq 5$, with peak currents not exceeding $2 \mathrm{~A}$ ). Technical details of the lock-in based scanning imaging set-up used to record plasmonic interferograms along the slit axes are given elsewhere ${ }^{17}$.

Optical constants. Thin-film calculations in gold-cobalt-gold multilayers were performed with experimentally obtained values of the dielectric susceptibility for gold $\epsilon_{\mathrm{Au}}=-24.8+1.5 i$, for air $\epsilon_{\mathrm{Air}}=1$ and tensor components for cobalt $\epsilon_{x x}=-17.1+24.2 i, Q=i \epsilon_{y z} / \epsilon_{x x}=0.0345+0.01 i$ at $\lambda=808 \mathrm{~nm}$. As $\left|\epsilon_{\mathrm{Au}}\right| \gg 1$ the skin depth for the surface plasmon $\delta_{\text {skin }}=(\lambda / 4 \pi) \operatorname{Im}\left(\sqrt{1+\epsilon_{A u}} / \epsilon_{A u}\right)$ is nearly identical to that for a plane electromagnetic wave under normal incidence $\delta_{\text {skin }}=(\lambda / 4 \pi) \operatorname{Im}\left(1 / \sqrt{ } \epsilon_{\Lambda u}\right)$

\section{References}

1. Barnes, W. L., Dereux, A. \& Ebbesen, T. W. Surface plasmon subwavelength optics. Nature 424, 824-830 (2003)

2. Genet, C. \& Ebbesen, T. W. Light in tiny holes. Nature 445, 39-46 (2007).

3. Ozbay, E. Plasmonics: merging photonics and electronics at nanoscale dimensions. Science 311, 189-193 (2008)

4. Atwater, H. A. The promise of plasmonics. Sci. Am. 296, 56-63 (2008).

5. Altewischer, E., van Exter, M. P. \& Woerdman, J. P. Plasmon-assisted transmission of entangled photons. Nature 418, 304-306 (2002).

6. Bozhevolnyi, S. I., Volkov, V. S., Devaux, E., Laluet, J. Y, \& Ebbesen, T. W. Channel plasmon subwavelength waveguide components including interferometers and ring resonators. Nature 440, 508-511 (2006).

7. Kubo, A. et al. Femtosecond imaging of surface plasmon dynamics in a nanostructured silver film. Nano Lett. 5, 1123-1127 (2005).

8. Krasavin, A. V. \& Zheludev, N. I. Active plasmonics: controlling signals in $\mathrm{Au} / \mathrm{Ga}$ waveguide using nanoscale structural transformations. Appl. Phys. Lett. 84, 1416-1418 (2004).

9. Pacifici, D., Lezec, H. J. \& Atwater, H. A. All-optical modulation by plasmonic excitation of CdSe quantum dots. Nature Photon. 1, 402-406 (2007).

10. Fedutik, Y., Temnov, V. V., Schöps, O., Woggon, U. \& Artemyev, M. V. Exciton-plasmon-photon conversion in plasmonic nanostructures. Phys. Rev. Lett. 99, 136802 (2007).

11. Akimov, A. V. et al. Generation of single optical plasmons in metallic nanowires coupled to quantum dots. Nature 450, 402-406 (2007).

12. Dicken, M. J. et al. Electrooptic modulation in thin film barium titanate plasmonic interferometers. Nano Lett. 8, 4048-4052 (2008)

13. Koller, D. M. et al. Organic plasmon-emitting diode. Nature Photon. 2, 684-687 (2008)

14. Palomba, S. \& Novotny, L. Nonlinear excitation of surface plasmon polaritons by four-wave mixing. Phys. Rev. Lett. 101, 056802 (2008).

15. Piredda, G., Smith, D. D., Wendling, B. \& Boyd, R. W. Nonlinear optical properties of a gold-silica composite with high gold fill fraction and the sign change of its nonlinear absorption. J. Opt. Soc. Am. B 25, 945-950 (2008).

16. MacDonald, K. F., Samson, Z. L., Stockman, M. I. \& Zheludev, N. I. Ultrafast active plasmonics. Nature Photon. 3, 55-58 (2008).

17. Temnov, V. V. et al. Femtosecond surface plasmon interferometry. Opt. Express 17, 8423-8432 (2009).

18. Stanciu, C. D. et al. All-optical magnetic recording with circularly polarized light. Phys. Rev. Lett. 99, 047601 (2007).

19. Wallis, R. F. Surface magnetoplasmons on semiconductors. In Boardman, A. D. (ed.) Electromagnetic Surface Modes, Ch. 15, 575-631 (John Wiley \& Sons, 1982). 20. Gonzalez-Diaz, J. B. et al. Surface-magnetoplasmon nonreciprocity effects in noble-metal/ferromagnetic heterostructures. Phys. Rev. B 76, 153402 (2007).

21. Hermann, C. et al. Surface-enhanced magneto-optics in metallic multilayer films. Phys. Rev. B 64, 235422 (2001).

22. Gonzalez-Diaz, J. B. et al. Enhanced magneto-optics and size effects in ferromagnetic nanowire arrays. Adv. Mater. 19, 2643-2647 (2007).

23. Gonzalez-Diaz, J. B. et al. Plasmonic $\mathrm{Au} / \mathrm{Co} / \mathrm{Au}$ nanosandwiches with enhanced magneto-optical activity. Small 4, 202-205 (2008).

24. Sepulveda, B., Lechuga, L. M. \& Armelles, G. Magnetooptic effects in surfaceplasmon-polaritons slab waveguides. J. Lightwave Technol. 24, 945-955 (2006).

25. Sepulveda, B., Calle, A., Lechuga, L. \& Armelles, G. Highly sensitive detection of biomolecules with the magneto-optic surface-plasmon-resonance sensor. Opt. Lett. 31, 1085-1087 (2006)

26. Gay, G. et al. The response of nanostructured surfaces in the near field. Nature Phys. 2, 262-267 (2006) 
27. Temnov, V. V., Woggon, U., Dintinger, J., Devaux, E. \& Ebbesen, T. W. Surface plasmon interferometry: measuring group velocity of surface plasmons. Opt. Lett. 32, 1235-1237 (2007).

28. Hillebrands, B. \& Fassbender, J. Applied physics: ultrafast magnetic switching. Nature 418, 493-495 (2002)

29. Kimel, A. V. et al. Ultrafast non-thermal control of magnetization by instantaneous photomagnetic pulses. Nature 435, 655-657 (2004)

30. Belotelov, V. I., Doskolovich, L. L. \& Zvezdin, A. K. Extraordinary magneto-optical effects and transmission through metal-dielectric plasmonic systems. Phys. Rev. Lett. 98, 077401 (2007).

\section{Acknowledgements}

This work was supported by the European Network of Excellence PhOREMOST, EU (NMP3-SL-2008-214107-Nanomagma), Spanish MICINN ("FUNCOAT' CONSOLIDER INGENIO 2010 CSD2008-00023 and 'MAGPLAS' MAT2008-06765-C02-01/NAN) CM ('NANOMAGNET' S-0505/MAT/0194, 'MICROSERES' S-0505/TIC/0191), The
German Research Foundation (DFG TE770/1). We also thank ].L. Costa-Krämer for the Transverse Kerr loop measurement and K. Nelson for stimulating discussions.

\section{Author contributions}

V.T., U.W., G.A. and A.C. wrote the proposal. V.T., U.W., G.A., A.C., A.G.M. and J.M.G.M. conceived and designed the experiments. G.A., A.C., A.G.M., J.M.G.M., T.T., A.L. and R.B. prepared and characterized the samples. A.C., D.G., A.G.M., T.T, A.L. and R.B. contributed materials and analysis tools. V.T. and G.A. performed the measurements and analysed the data. G.A., A.G.M., D.G. and V.T. carried out theoretical calculations. All authors wrote the manuscript.

\section{Additional information}

The authors declare no competing financial interests. Supplementary information accompanies this paper at www.nature.com/naturephotonics. Reprints and permission information is available online at http:/npg.nature.com/reprintsandpermissions/. Correspondence and requests for materials should be addressed to V.V.T 